\title{
Complexities in Assessing Structural Health of Civil Infrastructures
}

\author{
Abdullah Al-Hussein ${ }^{1}$ and Achintya Haldar ${ }^{2}$ \\ ${ }^{1}$ Department of Civil Engineering, University of Basrah, Basrah, Iraq \\ ${ }^{2}$ Department of Civil Engineering and Engineering Mechanics, University of Arizona, Tucson, AZ 85721, USA
}

Correspondence should be addressed to Achintya Haldar; haldar@u.arizona.edu

Received 7 May 2017; Accepted 13 August 2017; Published 14 September 2017

Academic Editor: Dimitri Volchenkov

Copyright (C) 2017 Abdullah Al-Hussein and Achintya Haldar. This is an open access article distributed under the Creative Commons Attribution License, which permits unrestricted use, distribution, and reproduction in any medium, provided the original work is properly cited.

\begin{abstract}
The complexity in the health assessment of civil infrastructures, as it evolves over a long period of time, is briefly discussed. A simple problem can become very complex based on the current needs, sophistication required, and the technological advancements. To meet the current needs of locating defect spots and their severity accurately and efficiently, infrastructures are represented by finite elements. To increase the implementation potential, the stiffness parameters of all the elements are identified and tracked using only few noise-contaminated dynamic responses measured at small part of the infrastructure. To extract the required information, Kalman filter concept is integrated with other numerical schemes. An unscented Kalman filter (UKF) concept is developed for highly nonlinear dynamic systems. It is denoted as 3D UKF-UI-WGI. The basic UKF concept is improved in several ways. Instead of using one long duration time-history in one global iteration, very short duration time-histories and multiple global iterations with weight factors are used to locate the defect spot more accurately and efficiently. The capabilities of the procedure are demonstrated with the help of two informative examples. The proposed procedure is much superior to the extended Kalman filter-based procedures developed by the team earlier.
\end{abstract}

\section{Introduction}

The complexity concept is very difficult to define for engineering applications. Considering practical necessity, the users often initially attempt to solve a problem using common sense. However, the solution strategy evolves over a very long period of time. It is not uncommon to find that the initial solution strategy was developed without using any mathematics-based concept. The same basic concept is being developed at present using the current state-of-theart advanced mathematical concepts embedded in highly powerful computational frame work. To assess health of an earthen utensil, our forefather tapped it and listened to the sound it produced. The world communities at present are trying to assess the health of civil infrastructures to help maintain our way of life. Some of these infrastructures were designed a long time ago and their design life may have expired. They need to be replaced; however, we do not have resources to replace them. Their design lives need to be extended. One attractive economical approach has attracted the attention of the profession is to inspect them as comprehensively as possible to identify the location, type, and severity of defects if any and then repair them in the most cost effective way to bring them back to the original state when initially designed.

Because of its relevance and importance, the structural health assessment (SHA) of civil infrastructures has attracted multidisciplinary research interest from all over the world. The basic health assessment problem of earthen utensils has now extended to infrastructures. As the health assessment concept started maturing over thousands of years, the users are now demanding different types of capabilities. Essentially, a simple concept has become very complex to satisfy the current needs. Similar challenges are faced by physicians to assess human health. They now have access to numerous equipment and test protocols with various degrees of sophistication. They need to use them very judiciously using information on cost and benefit. 
Looking back to the chronology of events for the development of SHA techniques for civil infrastructures, the engineering profession also followed strategies similar to the physicians. To identify the location, type, and severity of defects in large infrastructures, it is necessary to represent them in mathematical or algorithmic form, for example, representing them by finite elements. By tracking the structural properties of the elements and comparing them with the previous values if available from past inspections or changes from the original values mentioned in design drawings, deviation from the expected values, or variation with other similar elements, the location and the severity of defect in elements can be assessed. This model-based advancement replaced the non-model-based approaches used to check health of earthen utensils. The engineers also realized that to assess the current health, structural behavior at the time of the inspection needs to be used. This requirement adds another layer of complexity. Because of its simplicity, the profession started measuring responses by exciting the structures statically. Although it is relatively simple, it has major deficiencies [1-3] and may not be an attractive option. Measuring responses by exciting the structures dynamically removes some of the deficiencies in static approaches; it requires dynamic responses to be measured at all dynamic degrees of freedom (DDOFs) of infrastructures. It will be practically impossible to instrument large civil infrastructures. Response information may be measured at a limited number of DDOFs. To add fuel to the fire, dynamic responses are always expected to be noisecontaminated and this information needs to be mitigated appropriately.

Dynamic response information can be treated in the frequency or time domain. Since a large number of modes are difficult to evaluate [4] and the presence of defects may force the structure behave nonlinearly, the frequency domain approach may not be appropriate. Although it is relatively complicated but efficient in mitigating uncertainty related issues in the measured dynamic responses, the time domain approach has attracted the attention of the research team of the University of Arizona. Time domain approaches will be discussed in this paper.

The authors attempted to discuss above how basic SHA methods evolved with time and various levels of complexities were introduced considering the practical necessity, improving predictability, and implementation potential. The team first attempted to identify a defect in a plate in the form of a notch $[5,6]$ both experimentally and analytically. This exposed the team to a new reality. In addition to measuring dynamic responses, the dynamically applied excitation force also needs to be measured. It is well known to the profession that outside the controlled laboratory environment, measuring the excitation information in field conditions can be very expensive and the presence of noise can be beyond the control of an inspector. During this phase of investigation, the research team decided to use a system identification (SI) concept as the major building block. It has three basic components: excitation, dynamic responses, and the system to be identified. By measuring excitation and response information, the unknown system parameters can be identified to assess structural health. If the excitation information is ignored, then two of the three components are unknown and the SI process adds another layer of complexity.

For the wider applications and ease of implementation, the authors and their team members decided to identify a system without measuring excitation information. Initially, the team proposed a concept known as Iterative LeastSquares with Unknown Input (ILS-UI) [7-9] to identify a system using only noise-contaminated response information measured at all dynamic degrees of freedoms (DDOFs). At this stage, the team observed that it may be uneconomical and impractical to instrument large infrastructures and measure responses at all DDOFs. Responses may be measured only at a part of large infrastructures. For SI with limited response information, Kalman filter (KF) concept $[10,11]$ can be used. It is used by electrical engineers for signal processing. However, to implement it, the excitation information and initial unknown state vector (which includes the state of elements, the final outcome of an inspection) must be known. These requirements will invalidate the team previous work of developing methods with unknown input. Since limited response information is also a practical necessity, the team decided to propose a two-stage approach by combining ILS$\mathrm{UI}$ and KF approaches. At this stage, the works crossed the engineering disciplinary boundaries. Since defects in infrastructures can make them behave nonlinearly, the team decided to use piecewise linear or Extended KF concept or (EKF). The team proposed several methods using the ILSEKF-UI concept for two-dimensional (2D) $[12,13]$ and threedimensional (3D) [14] infrastructures. Most recently, the authors observed another limitation of EKF-based concept. The derivative process used in EKF can introduce significant error and the algorithm may not converge or converge to incorrect values and may incorrectly predict the current state of elements. This prompted the authors to propose another alternative of using Unscented KF or UKF $[15,16]$. During the development of the UKF concept, they realized that since it would be an iterative process, the use of weighted global iteration (WGI) can improve the efficiency and accuracy of the algorithm. Initially, they proposed a novel concept commonly known as UKF-UI-WGI for 2D infrastructures [17, 18]. Most recently, they extended the concept to 3D infrastructures and denoted it as 3D UKF-UI-WGI procedure [19].

As briefly mentioned earlier, the finite elements are utilized to represent a structure and defective elements can be identified using the proposed procedure. In some cases, the inspection of a defective element may not be economical if the defect spot within the element can be located effortlessly. Such desirable capability of 3D UKF-UI-WGI is demonstrated in this paper. The authors also conclusively demonstrated its superiority over EKF-based procedures developed by the other research team members in the past.

The above discussions clearly indicate how a simple problem can become very complex crossing engineering disciplinary boundaries and boundaries between science and engineering. It is not practical to give mathematical details of all the concepts developed by the authors and their team members in this paper. The two-stage approach used in developing 3D UKF-UI-WGI is briefly discussed and its 
capabilities in assessing structural health are showcased with the help of informative examples.

\section{Concept of the Two-Stage Approach in 3D UKF-UI-WGI}

The main purpose of the 3D UKF-UI-WGI approach is to assess health of civil infrastructures using minimum number of dynamic response information. The only available information is few noise-contaminated response time-histories measured at a small part of infrastructure. The unknowns are the input excitation time-history, response time-histories not measured at the other part of the structure, the stiffness parameters of all the members or the elements in the finite element representation, and the damping coefficients required to satisfy the dynamic governing equations of motion of the system. Therefore, the number of unknowns is expected to be much higher than the known variables and the problem needs to be addressed in a very systematic way. To use any KF-based algorithm, its two basic requirements must be addressed first. A two-stage approach is proposed to mitigate the problem. The main purpose of stage 1 is to identify the excitation time-history and the unknown state vector. To generate the required information, a substructure concept is introduced in stage 1. Considering the locations of the excitation and the measured response information, a substructure can be defined as discussed in more detail in $[3,13]$. Since the basic algorithm is finite element based, the damping is represented by two Rayleigh coefficients. Since responses at all DDOFs are measured in the substructure, the 3D ILS-UI procedure can be used to identify the unknown input excitation time-history, stiffness parameters of all the elements in the substructure, and two Rayleigh damping coefficients. Since the stiffness of structural elements in the substructure and the rest of the structure is expected to be similar in real infrastructures, the identified stiffness information of the substructure can be judiciously used to generate the initial information of the state vector of the whole structure. A substructure is expected to consist of a beam and a column element. Since the stiffness parameters of other beams and columns are expected to be similar, they can be assigned the corresponding stiffness parameters to generate the initial state vector for the whole infrastructure. The information generated in stage 1 will satisfy all the requirements to implement any KF-based algorithm. Thus, in stage 2, the UKF concept can be introduced to identify the stiffness parameters of all the elements in the infrastructures. Essentially, using only the response time-histories measured in the substructure, the stiffness properties of all the elements in the infrastructures can be obtained to assess health, as discussed earlier, using the 3D UKF-WGI procedure satisfying the basic objective of the study. The mathematical concepts of 3D UKF-WGI are briefly discussed below.

\section{Mathematical Formulation of the 3D UKF-UI-WGI Procedure}

As mentioned earlier, 3D UKF-UI-WGI is a two-stage procedure, as discussed below.

3.1. Stage 1: Formulation of 3D ILS-UI. A structural dynamics problem is typically formulated using the following secondorder differential equation:

$$
\mathbf{M X}(t)+\mathbf{C} \dot{\mathbf{X}}(t)+\mathbf{K X}(t)=\mathbf{f}(t),
$$

where $\mathbf{M}, \mathbf{C}$, and $\mathbf{K}$ are mass, damping, and stiffness matrix, respectively, of the structure; $\ddot{\mathbf{X}}(t), \dot{\mathbf{X}}(t)$, and $\mathbf{X}(t)$ are acceleration, velocity, and displacement vector, respectively; and $\mathbf{f}(t)$ is the excitation force. The efficiency of the SI procedures improves using the two mass and stiffness proportional Rayleigh-type damping coefficients.

The mass and stiffness matrices of the $i$ th frame element of a 3D infrastructure can be expressed as [20]

$$
\overline{\mathbf{M}}_{i}=\frac{\bar{m}_{i} L_{i}}{420}\left[\begin{array}{cccccccccccc}
140 & 0 & 0 & 0 & 0 & 0 & 70 & 0 & 0 & 0 & 0 & 0 \\
0 & 156 & 0 & 0 & 0 & 22 L_{i} & 0 & 54 & 0 & 0 & 0 & -13 L_{i} \\
0 & 0 & 156 & 0 & -22 L_{i} & 0 & 0 & 0 & 54 & 0 & 13 L_{i} & 0 \\
0 & 0 & 0 & 140 a_{i} & 0 & 0 & 0 & 0 & 0 & 70 a_{i} & 0 & 0 \\
0 & 0 & -22 L_{i} & 0 & 4 L_{i}^{2} & 0 & 0 & 0 & -13 L_{i} & 0 & -3 L_{i}^{2} & 0 \\
0 & 22 L_{i} & 0 & 0 & 0 & 4 L_{i}^{2} & 0 & 13 L_{i} & 0 & 0 & 0 & -3 L_{i}^{2} \\
70 & 0 & 0 & 0 & 0 & 0 & 140 & 0 & 0 & 0 & 0 & 0 \\
0 & 54 & 0 & 0 & 0 & 13 L_{i} & 0 & 156 & 0 & 0 & 0 & -22 L_{i} \\
0 & 0 & 54 & 0 & -13 L_{i} & 0 & 0 & 0 & 156 & 0 & 22 L_{i} & 0 \\
0 & 0 & 0 & 70 a_{i} & 0 & 0 & 0 & 0 & 0 & 140 a_{i} & 0 & 0 \\
0 & 0 & 13 L_{i} & 0 & -3 L_{i}^{2} & 0 & 0 & 0 & 22 L_{i} & 0 & 4 L_{i}^{2} & 0 \\
0 & -13 L_{i} & 0 & 0 & 0 & -3 L_{i}^{2} & 0 & -22 L_{i} & 0 & 0 & 0 & 4 L_{i}^{2}
\end{array}\right] \text {, }
$$




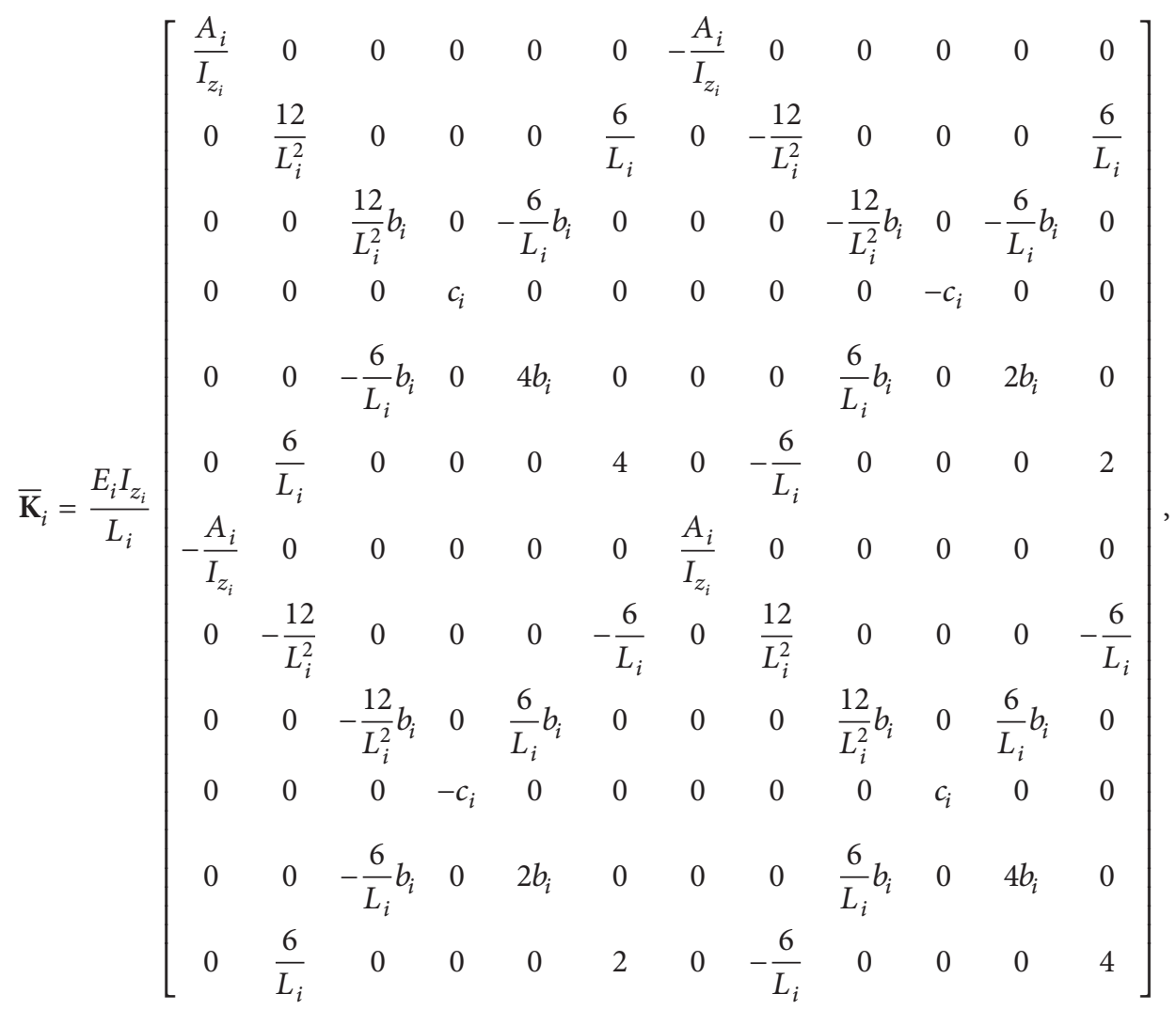

where

$$
\begin{aligned}
a_{i} & =\frac{I_{\bar{m}_{i}}}{\bar{m}_{i}}, \\
b_{i} & =\frac{I_{y_{i}}}{I_{z_{i}}} \\
c_{i} & =\frac{J_{i}}{\left[2(1+v) I_{z_{i}}\right]},
\end{aligned}
$$

where $L_{i}$ and $A_{i}$ are the length and cross-sectional area, respectively, of the $i$ th element; $I_{z_{i}}$ and $I_{y_{i}}$ are moment of inertia of the cross-section with respect to the major and minor principal axes, respectively; $I_{\bar{m}_{i}}$ and $J_{i}$ are polar mass moment of inertia per unit length and torsional moment of inertia, respectively; and $E_{i}, \bar{m}_{i}$, and $v$ are modulus of elasticity, mass per unit length, and Poisson's ratio of the material, respectively.

In stage 1, the governing differential equation of motion of the key node (the point of application of the excitation force) in the substructure is only needed to be considered and it can be expressed in the presence of Rayleigh damping as

$$
\begin{aligned}
& \mathbf{M}_{\mathrm{kn}, \mathrm{sub}} \ddot{\mathbf{X}}_{\mathrm{sub}}(t)+\left(a \mathbf{M}_{\mathrm{kn}, \mathrm{sub}}+b \mathbf{K}_{\mathrm{kn}, \mathrm{sub}}\right) \dot{\mathbf{X}}_{\mathrm{sub}}(t) \\
& +\mathbf{K}_{\mathrm{kn}, \mathrm{sub}} \mathbf{X}_{\text {sub }}(t)=\mathbf{f}_{\mathrm{kn}}(t),
\end{aligned}
$$

where $a$ and $b$ are mass and stiffness proportional Rayleigh damping coefficients, respectively; and $\mathrm{kn}$ and sub stand for

key node and substructure, respectively. The subscripts kn and sub represent vector with dimension of $\mathrm{kn} \times 1$ and sub $\times 1$, respectively, and $\mathrm{kn}$, sub represents a matrix with dimension of $\mathrm{kn} \times$ sub.

Equation (4) can be rearranged in the matrix form as

$$
\mathbf{A}_{\mathrm{kn}, \mathrm{sub}}(t) \mathbf{P}_{\mathrm{sub}}=\mathbf{F}_{\mathrm{kn}}(t),
$$

where $\mathbf{A}_{\mathrm{kn}, \text { sub }}(t)$ is a matrix including the displacement and velocity responses at time point $t$; $\mathbf{P}_{\text {sub }}$ is a vector of the unknown parameters (stiffness parameters and two damping coefficients); and $\mathbf{F}_{\mathrm{kn}}(t)$ is a vector comprised of the unknown input excitation and inertia forces at time point $t$. The stiffness parameter of the $i$ th element is represented as $E_{i} I_{z_{i}} / L_{i}$, in this study. To identify the unknown parameters of the substructure, the responses need to be measured for $q$ time points.

Equation (5) can be rewritten for $q$ time points as

$$
\mathbf{A}_{q \cdot \mathrm{kn}, \mathrm{sub}} \mathbf{P}_{\text {sub }}=\mathbf{F}_{q \cdot \mathrm{kn}},
$$

where $\mathbf{F}_{q \cdot k n}$ is a vector with dimension of $(q \times \mathrm{kn}) \times 1$ and $\mathbf{A}_{q \cdot \mathrm{kn}, \text { sub }}$ is a matrix with dimension of $(q \times \mathrm{kn}) \times$ sub.

Since the number of equations in (6) is more than the number of the unknowns, the least-squares technique is used to solve for the unknown parameters in $\mathbf{P}_{\text {sub }}$ vector as

$$
\mathbf{P}_{\text {sub }}=\left(\mathbf{A}_{\text {sub }, q \cdot \mathrm{kn}}^{T} \mathbf{A}_{q \cdot \mathrm{kn}, \mathrm{sub}}\right)^{-1} \mathbf{A}_{\text {sub }, q \cdot \mathrm{kn}}^{T} \mathbf{F}_{q \cdot \mathrm{kn}} .
$$


Equation (7) has unknowns on both sides. In the left side, vector $\mathbf{P}_{\text {sub }}$ is unknown and, in the right side, vector $\mathbf{F}_{q \cdot \mathrm{kn}}$ includes unknown inertia force. Therefore, to initiate the iteration process for solving the unknown parameters in $\mathbf{P}_{\text {sub }}$ vector, the unknown excitation forces are assumed to be zero at all time points, as suggested by Wang and Haldar [7] and Katkhuda et al. [9]. The iteration process continues until a predetermined convergence tolerance level $\left(\varepsilon_{f}\right)$ on the input excitation at all time points is obtained. The convergence requires $\left|\mathbf{f}_{\mathrm{kn}}^{i+1}-\mathbf{f}_{\mathrm{kn}}^{i}\right|<\varepsilon_{f}$ where $\mathbf{f}_{\mathrm{kn}}^{i+1}$ and $\mathbf{f}_{\mathrm{kn}}^{i}$ are the forces estimated in two consecutive time steps. After the completion of the iteration process of 3D ILS-UI, the unknown input excitation time-history, stiffness parameters of all the elements in the substructure, and the two damping coefficients will be available.

3.2. Stage 2: Formulation of $3 D$ UKF-WGI. The general dynamic system can be expressed by a nonlinear continuous state-space equation as

$$
\dot{\mathbf{X}}_{t}=f\left(\mathbf{X}_{t}, t\right),
$$

and the nonlinear discrete measurement equation at time point $t=k \Delta t$ as

$$
\mathbf{Y}_{k}=h\left(\mathbf{X}_{k}, t\right)+\mathbf{V}_{k}
$$

where $\mathbf{X}_{t}$ is state variable vector at time point $t$; $\dot{\mathbf{X}}_{t}$ is derivative of the state variable vector with respect to time; $\mathbf{Y}_{k}$ is the measurement vector at $t=k \Delta t$; $\mathbf{V}_{k}$ is the zero mean Gaussian measurement noise vector with the corresponding covariance matrix $\mathbf{R}_{k}$; and $f$ and $h$ are the nonlinear functions.

The state variable vector is augmented in order to include the stiffness parameters to be identified, that is,

$$
\mathbf{X}_{t}=\left[\begin{array}{c}
\mathbf{X}(t) \\
\dot{\mathbf{X}}(t) \\
\widetilde{\mathbf{K}}
\end{array}\right],
$$

where $\mathbf{X}(t)$ and $\dot{\mathbf{X}}(t)$ denote the displacement and velocity vectors, respectively; and $\widetilde{\mathbf{K}}$ contains the unknown stiffness parameter vector.

After the initial conditions of variables in the state vector and corresponding uncertainty are assigned appropriately, the UKF filtering process can be initiated to estimate the state mean vector and its error covariance matrix at time step $(k+1)$.

At time point $k$, the sigma points, which are distributed symmetrically around the mean, are computed by

$$
\boldsymbol{\chi}_{k \mid k}=\left[\begin{array}{c}
\widehat{\mathbf{X}}_{k \mid k} \\
\widehat{\mathbf{X}}_{k \mid k}+\sqrt{\left(\lambda+l_{x}\right) \mathbf{P}_{k \mid k}} \\
\widehat{\mathbf{X}}_{k \mid k}-\sqrt{\left(\lambda+l_{x}\right) \mathbf{P}_{k \mid k}}
\end{array}\right],
$$

where $\lambda$ is a scaling parameter which is defined by

$$
\lambda=\alpha^{2}\left(l_{x}+\kappa\right)-l_{x}
$$

where $\mathbf{P}_{k \mid k}$ is the corresponding covariance vector; $\alpha$ and $\kappa$ are the KF parameters; and $l_{x}$ is the dimension of the augmented state variable vector.

Once the $\boldsymbol{\chi}_{k \mid k}$ is computed, the predicted state variable vector $\widehat{\mathbf{X}}_{k+1 \mid k}$ and its predicted covariance matrix $\mathbf{P}_{k+1 \mid k}$ are computed as

$$
\begin{aligned}
\chi_{k+1 \mid k} & =\chi_{k \mid k}+\int_{k \Delta t}^{(k+1) \Delta t} f\left(\mathbf{X}_{t}, t\right) d t \quad i=0, \ldots, 2 l_{x}, \\
\widehat{\mathbf{X}}_{k+1 \mid k} & =\sum_{i=0}^{2 l_{x}} W_{i}^{m} \chi_{i, k+1 \mid k}, \\
\mathbf{P}_{k+1 \mid k} & =\sum_{i=0}^{2 l_{x}} W_{i}^{c}\left(\chi_{i, k+1 \mid k}-\widehat{\mathbf{X}}_{k+1 \mid k}\right)\left(\chi_{i, k+1 \mid k}-\widehat{\mathbf{X}}_{k+1 \mid k}\right)^{T},
\end{aligned}
$$

where $W_{i}^{m}$ is the weight for the mean and $W_{i}^{c}$ is the weight for the covariance given by

$$
\begin{aligned}
W_{0}^{m} & =\frac{\lambda}{\lambda+l_{x}}, \\
W_{0}^{c} & =\frac{\lambda}{\lambda+l_{x}}+\left(1-\alpha^{2}+\beta\right), \\
W_{i}^{m} & =W_{i}^{c}=\frac{1}{2\left(\lambda+l_{x}\right)} \quad i=1, \ldots, 2 l_{x},
\end{aligned}
$$

where $\beta$ is used to incorporate prior knowledge of the distribution of $\mathbf{X}$ (for Gaussian distributions, $\beta=2$ is optimal).

The predicted measurement vector $\widehat{\mathbf{Y}}_{k+1 \mid k}$ and its error covariance matrix $\mathbf{P}_{k+1 \mid k}^{Y Y}$ can be expressed as

$$
\begin{aligned}
\widehat{\mathbf{Y}}_{k+1 \mid k} & =\mathbf{H} \widehat{\mathbf{X}}_{k+1 \mid k}, \\
\mathbf{P}_{k+1 \mid k}^{Y Y} & =\mathbf{H P}_{k+1 \mid k} \mathbf{H}^{T}+\mathbf{R}_{k+1} .
\end{aligned}
$$

Then, the measurement update equations are as follows:

$$
\begin{aligned}
\widehat{\mathbf{X}}_{k+1 \mid k+1} & =\widehat{\mathbf{X}}_{k+1 \mid k}+\mathbf{K}_{k+1}\left(\mathbf{Y}_{k+1}-\widehat{\mathbf{Y}}_{k+1 \mid k}\right), \\
\mathbf{P}_{k+1 \mid k+1} & =\mathbf{P}_{k+1 \mid k}-\mathbf{K}_{k+1} \mathbf{P}_{k+1 \mid k}^{Y Y} \mathbf{K}_{k+1}^{T},
\end{aligned}
$$

where $\mathbf{K}_{k+1}$ is the Kalman gain matrix at time step $k+1$, which can be expressed as

$$
\begin{aligned}
\mathbf{K}_{k+1} & =\mathbf{P}_{k+1 \mid k}^{X Y}\left(\mathbf{P}_{k+1 \mid k}^{Y Y}\right)^{-1}, \\
\mathbf{P}_{k+1 \mid k}^{X Y} & =\mathbf{P}_{k+1 \mid k} \mathbf{H}^{T} .
\end{aligned}
$$

Equations from (11) to (17) are the iteration process of the UKF algorithm between two successive time points, that is, between $k$ and $k+1$, and this process is generally denoted as a local iteration. The local iteration needs to be carried out for all $q$ time points and this process is called a global iteration. The classical UKF algorithm used only one global iteration with relatively long measured response time-histories. The long duration increases the possibility of excitations caused by unwanted sources beyond the control of inspectors in 


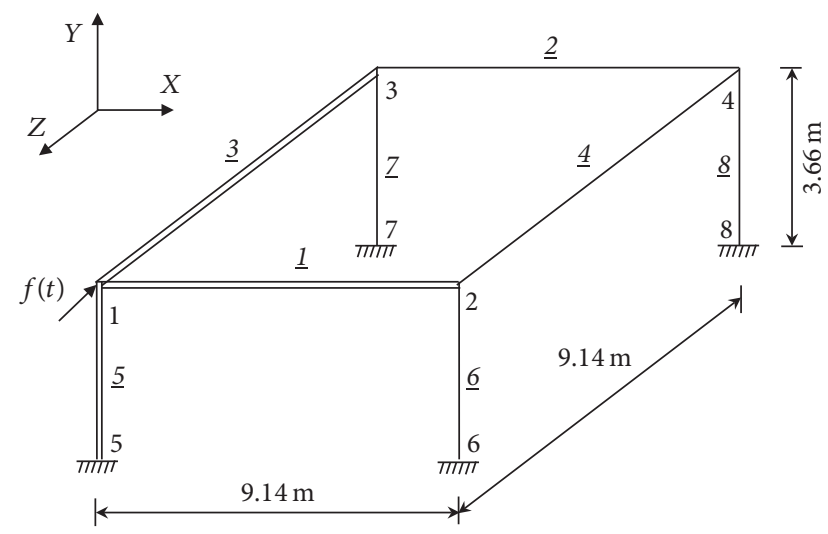

FIGURE 1: Finite element representation of 3D single-story frame.

an actual inspection. This additional source of noise or uncertainty may not help to identify large infrastructures [21]. To overcome this shortcoming, the authors proposed to use short duration response information but more than one global iteration. Since it is an iterative process, they introduced a weight factor [22] for each global iteration. The weight factor is used to amplify the error covariance matrix of stiffness parameters at the end of each global iteration to use it as the initial covariance matrix for the next global iteration. The UKF algorithm with weighted global iteration (WGI) gives optimal, stable, and convergent solution. However, using a large value of weight factor may compromise the convergence criteria. To address the issue, an objective function [22] is also introduced to be used if the algorithm diverges.

\section{Verification of the Procedure}

To demonstrate the capability of the procedure, the health assessments of two 3-D frames with different sizes are considered. The verification process considers the identification of the defective element and the severity of the defect. If a member is found to be defective, the algorithm also locates the defect spot more accurately within the defective member.

\subsection{Example 1: 3D Single-Story Frame}

4.1.1. Description of the Problem. The first example is a $3 \mathrm{D}$ single-story steel frame as shown in Figure 1. The frame has square base of sides $9.14 \mathrm{~m}$ and story height of $3.66 \mathrm{~m}$. The frame is made of Grade 50 steel members. The beams and the columns are made of $\mathrm{W} 21 \times 68$ sections and $\mathrm{W} 14 \times 61$ sections, respectively. The frame is represented by the finite element with 8 nodes and 8 elements, 4 beams and 4 columns, as shown in Figure 1.

Each node has six DDOFs, three translational and three rotational. The frame support at the base (nodes 5, 6, 7, and 8 ) is considered to be fixed. The first two natural frequencies of the frame are estimated to be $f_{1}=6.6539 \mathrm{~Hz}$ and $f_{2}=$ $8.2783 \mathrm{~Hz}$, respectively. Assuming damping to be the same for the first two natural modes and following procedure suggested in [23], the two Rayleigh damping coefficients, $a$ and $b$, are calculated to be 2.317787 and 0.001066 , respectively, for an equivalent modal damping of $5 \%$ (commonly used in the model codes in the US) of the critical.

The responses are generated analytically by applying a harmonic excitation, $f(t)=50 \sin (40 t)$, at node 1 along the $Z$-direction as shown in Figure 1. Commercial software ANSYS [24] is used for this purpose. The responses are recorded at 3 nodes $(1,2$, and 3$)$, at the interval $0.0001 \mathrm{~s}$ for a total duration of $0.63 \mathrm{~s}$. After the responses are obtained, the information on input excitation is completely ignored. Responses between $0.31 \mathrm{~s}$ and $0.63 \mathrm{~s}$ providing 3201 samples are used in the subsequent identification process. During an actual inspection, these responses will be measured by accelerometers. The configuration of the substructure for the frame is shown in Figure 1 with double lines. It has 3 elements $(1,3$, and 5$)$ and 4 nodes $(1,2,3$, and 5$)$. Since node 5 is fixed, the total number of DDOFs of the substructure is 18 .

4.1.2. Identification of Defect-Free Frame. Initially, the system identification of defect-free frame is considered. In stage 1, the $3 \mathrm{D}$ ILS-UI procedure is implemented to identify the substructure using only the responses at 18 DDOFs. The stiffness parameters of two beams and one column in the substructure, two Rayleigh damping coefficients, and the excitation time-history are identified accurately. The identified stiffness parameters and the error in identification are shown in columns (3) and (4), respectively, of Table 1(a). It can be observed that the error in the identification is very small. In stage 2, the $3 \mathrm{D}$ UKF-WGI procedure is used to identify the stiffness parameters of all the elements in the structure using the responses only at the substructure and the information generated in stage 1 . The identified stiffness parameters are summarized in Table 1(b). Since there are no significant changes between the actual and identified stiffness parameters, the frame can be considered as defect-free.

4.1.3. Identification of Defective Frame. The two defective states of the frame are considered in this section. In defect 1 , the web and flange thicknesses of beam 2 over the entire length are considered to be reduced by $20 \%$ of the defectfree value due to corrosion. This defect caused the reduction in the principal moment of inertia about the major axis $\left(I_{z}\right)$ by $22.15 \%$, principal moment of inertia about the minor axis $\left(I_{y}\right)$ by $20.05 \%$, and cross-sectional area $(A)$ by $20.74 \%$. In defect 2 , a less severe defect is considered. The web and flange thicknesses of beam 4 are considered to be corroded over a finite length. The reduction is $50 \%$ of defect-free value over a length of $20 \mathrm{~cm}$, located at a distance $50 \mathrm{~cm}$ from node 2 as shown in Figure 2. The loss of thicknesses will result in the reduction in the principal moment of inertia about the major axis $\left(I_{z}\right)$ by $52.14 \%$, principal moment of inertia about the minor axis $\left(I_{y}\right)$ by $50.07 \%$, and cross-sectional area $(A)$ by $50.47 \%$. Similar defect was considered in the laboratory investigation by other team members [25].

Using only the responses at the substructure, the stiffness parameters of three members, two Rayleigh damping coefficients, and input excitation time-history are identified 
TABLE 1: Stiffness parameter identification of 3D single-story frame.

(a) Identification of substructure using 3D ILS-UI

\begin{tabular}{lccccccc}
\hline \multirow{2}{*}{ Member } & $\begin{array}{c}\text { Actual }(k) \\
(\mathrm{kN}-\mathrm{m})\end{array}$ & \multicolumn{2}{c}{ Defect-free } & \multicolumn{2}{c}{ Defect 1 } & \multicolumn{2}{c}{ Defect 2 } \\
& Identified $(k)$ & Change $(\%)$ & Identified $(k)$ & Change $(\%)$ & Identified $(k)$ & Change $(\%)$ \\
\hline$(1)$ & $(2)$ & $(3)$ & $(4)$ & $(5)$ & $(6)$ & $(7)$ & 13475.8 \\
\hline$k_{1}$ & 13476.2 & 13475.8 & -0.003 & 13475.9 & -0.002 & -0.003 \\
$k_{3}$ & 13476.2 & 13473.9 & -0.017 & 13473.9 & -0.017 & 13473.9 & -0.017 \\
$k_{5}$ & 14552.9 & 14551.7 & -0.008 & 14551.7 & -0.008 & 14551.8 & -0.008 \\
\hline
\end{tabular}

(b) Identification of whole structure using 3D UKF-WGI

\begin{tabular}{|c|c|c|c|c|c|c|c|}
\hline \multirow{2}{*}{ Member } & \multirow{2}{*}{$\begin{array}{c}\text { Actual }(k) \\
(\mathrm{kN}-\mathrm{m})\end{array}$} & \multicolumn{2}{|c|}{ Defect-free } & \multicolumn{2}{|c|}{ Defect 1} & \multicolumn{2}{|c|}{ Defect 2} \\
\hline & & Identified $(k)$ & Change (\%) & Identified $(k)$ & Change (\%) & Identified $(k)$ & Change (\%) \\
\hline (1) & (2) & (3) & (4) & (5) & (6) & (7) & (8) \\
\hline$k_{1}$ & 13476 & 13471 & -0.04 & 13462 & -0.10 & 13349 & -0.95 \\
\hline$k_{2}$ & 13476 & 13471 & -0.04 & 10744 & -20.27 & 13655 & 1.32 \\
\hline$k_{3}$ & 13476 & 13473 & -0.02 & 13468 & -0.06 & 13471 & -0.04 \\
\hline$k_{4}$ & 13476 & 13475 & -0.01 & 13495 & 0.14 & 12901 & -4.26 \\
\hline$k_{5}$ & 14553 & 14550 & -0.02 & 14543 & -0.07 & 14554 & 0.01 \\
\hline$k_{6}$ & 14553 & 14551 & -0.02 & 14558 & 0.03 & 14546 & -0.05 \\
\hline$k_{7}$ & 14553 & 14548 & -0.04 & 14543 & -0.07 & 14471 & -0.56 \\
\hline$k_{8}$ & 14553 & 14547 & -0.04 & 14549 & -0.03 & 14514 & -0.27 \\
\hline
\end{tabular}

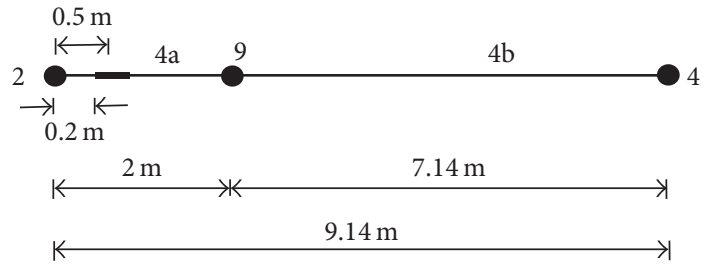

FIGURE 2: Defect 2 representation.

accurately for the two defective cases using the 3D ILS-UI procedure in stage 1 . The results of the identified stiffness parameter and the changes with respect to the actual values for defect 1 are summarized in columns (5) and (6), respectively, of Table 1(a). The similar information for defect 2 is summarized in columns (7) and (8), respectively, of Table 1(a). Then, using the same responses in the substructure and the information extracted from stage 1, the stiffness parameters of all the elements in the structure are identified for the two defective cases using the 3D UKF-WGI procedure in stage 2 . The identification results for defect 1 are shown in columns (5) and (6) of Table 1(b) and for defect 2 in columns (7) and (8) of Table 1(b). The results of defect 1 show that the maximum reduction in the identified stiffness parameters is $20.27 \%$ for member 2 . The procedure correctly identified the location and severity of the defect in the frame. The identification results of defect 2 demonstrate that the maximum reduction occurs in member 4 , indicating it contains the defect. The results also show that the reduction in the identified stiffness parameter for defect 2 is much less than that of defect 1 , indicating it contains a less sever defect. These damage scenarios clearly demonstrate that the 3D UKF-UIWGI method is capable of identifying the location and the severity of the small and large defects accurately.

The capability of the 3D UKF-UI-WGI procedure is further examined if it can identify the location of the defective spot more accurately in a defective member. To locate the defect spot more accurately, the identified defective member needs to be represented by more number of finite elements and then the segment with the largest reduction in the stiffness parameter can be identified. This additional step can be undertaken without conducting any new experiment. To illustrate the procedure, defective member 4 is represented by two elements in this example. A new node is introduced at a distance of $2 \mathrm{~m}$ from node 2 . In the finite element representation, the two elements are denoted as $4 \mathrm{a}$ and $4 \mathrm{~b}$, as shown in Figure 2. The identified stiffness parameters of the whole frame using responses at 18 DDOFs are shown in column (3) of Table 2. The stiffness parameter for element $4 \mathrm{a}$ is reduced by the maximum amount of $17.16 \%$, significantly more than other members. It is clear that element 4 a contains the defect. To identify the location of defective spot more accurately, the length of the element $4 \mathrm{a}$ can be reduced further and the frame can be identified again using 3D UKF-WGI. The process can be continued until the accuracy requirement of locating the defect spot is satisfied. Since length of the segments in the defective element is reduced, the stiffness parameter of these added segments increases accordingly. This example confirms that 3D UKF-UI-WGI can detect a defective element and if necessary, the defect spot more accurately in a defective element. 
TABLE 2: Identification of defective spot within the defective member 4 using 3D UKF-WGI.

\begin{tabular}{lccc}
\hline Member & $\begin{array}{c}\text { Actual }(k) \\
(\mathrm{kN}-\mathrm{m})\end{array}$ & $\begin{array}{c}\text { Identified } \\
(k)\end{array}$ & $\begin{array}{c}\text { Change } \\
(\%)\end{array}$ \\
\hline$(1)$ & $(2)$ & $(3)$ & $(4)$ \\
\hline$k_{1}$ & 13476 & 13585 & 0.81 \\
$k_{2}$ & 13476 & 13351 & -0.93 \\
$k_{3}$ & 13476 & 13471 & -0.04 \\
$k_{4 \mathrm{a}}$ & 61586 & 51021 & -17.16 \\
$k_{4 \mathrm{~b}}$ & 17251 & 18056 & 4.67 \\
$k_{5}$ & 14553 & 14565 & 0.08 \\
$k_{6}$ & 14553 & 14539 & -0.09 \\
$k_{7}$ & 14553 & 14543 & -0.07 \\
$k_{8}$ & 14553 & 14519 & -0.23 \\
\hline
\end{tabular}

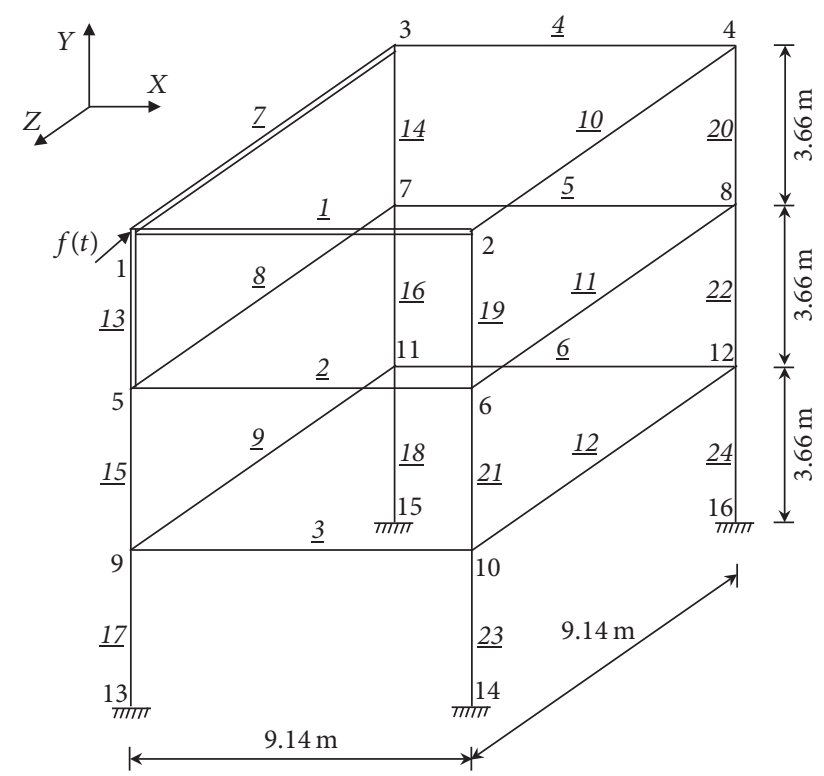

Figure 3: Finite element representation of 3D three-story frame.

4.2. Example 2: $3 D$ Three-Story Frame. The aim of this example is to compare the capability of the 3D UKF-UIWGI procedure with the 3D EKF-based procedure developed earlier by Das and Haldar [14]. The selection of 3D threestory frame presented in this example for comparison is for two reasons: (1) results of the identification of the frame using the EKF-based procedure are available [2] and (2) the differences in the identified results between UKF and EKF-based procedures become very clear for large structural systems.

4.2.1. Description of the Problem. A 3D three-story frame with square base of sides $9.14 \mathrm{~m}$ and floor to floor height of $3.66 \mathrm{~m}$, as shown in Figure 3, is considered. The beams and columns are made of W21 $\times 68$ and W14 $\times 61$ sections, respectively, of Grade 50 steel. In the finite element representation, the frame is represented by 16 nodes, denoted by numbers in regular typescript, and 24 elements, denoted by numbers in italic typescript with underline. All the elements are considered to be 3D beam elements with rigid connections. The support condition at the base of the frame (nodes 13, 14,15 , and 16) is considered to be fixed. Therefore, the total number of DDOFs for the frame is 72 . The actual stiffness parameters are calculated to be $13476 \mathrm{kN}-\mathrm{m}$ and $14553 \mathrm{kN}$ $\mathrm{m}$ for a typical beam and column, respectively. First two natural frequencies of the frame are estimated to be $f_{1}=$ $2.7229 \mathrm{~Hz}$ and $f_{2}=3.5717 \mathrm{~Hz}$, respectively. Following the procedure described in Clough and Penzien [23], the two Rayleigh damping coefficients $a$ and $b$ are calculated to be 0.970775 and 0.002528 , respectively, for an equivalent modal damping of $5 \%$ of the critical for the first two modes.

The numerical simulation of the frame in ANSYS software [24] is used to generate the responses numerically. The frame is excited by a force of $f(t)=45 \sin (20 t)$, at node 1 along the $Z$-direction, as shown in Figure 3. The responses are recorded only at 4 nodes $(1,2,3$, and 5$)$, at the interval $0.0001 \mathrm{~s}$ for a total duration of $0.63 \mathrm{~s}$. After the responses are obtained, the information on input excitation is completely ignored. Responses between $0.31 \mathrm{~s}$ and $0.63 \mathrm{~s}$ providing 3201 samples are used in the subsequent identification process. The substructure used in the subsequent health assessment process is shown in double lines in Figure 3. It comprises 3 elements $(1,7$, and 13$)$ and 4 nodes $(1,2,3$, and 5$)$.

4.2.2. Identification of the Frame. The capabilities of UKF and EKF procedures to identify the location and severity of defect are compared in this example. Member 2, which connects nodes 5 and 6 , is considered to be corroded. The thicknesses of web and flange of member 2 are reduced by $30 \%$. Accordingly, the principal moment of inertia about major axis $\left(I_{z}\right)$, principal moment of inertia about minor axis $\left(I_{y}\right)$ and cross-sectional areas $(A)$ by $32.26 \%, 30.07 \%$, and $30.65 \%$, respectively. Without knowing the location and severity of defect and using only responses at 4 nodes (24 DDOFs), the stiffness parameters of three members in the substructure, the input excitation time-history, and the damping coefficients are identified in stage 1 . Then, using only the responses at 24 DDOFs in the substructure and the required information extracted from stage 1 , the stiffness parameters of all members in the frame are identified using 3D UKF-WGI as summarized in Table 3 columns (3) and (4). It can be noticed that the location and severity of defect are identified accurately and the identification errors in the other defect-free members in the frame are small. For comparison, the results of EKF-based procedure with response at 24 DDOFs are presented, as shown in Table 3 columns (5) and (6). It can be seen that the errors in the identification of defect-free members are large. For example, the stiffness reduction of member 3 is $14.45 \%$, erroneously indicating that it is a defective member. Therefore, it can be concluded that the EKF-based procedure failed to identify the frame. Das [2] showed that the EKF-based procedure could identify the frame using responses measured at least at 27 DDOFs, as shown in Table 3 columns (7) and (8).

The examples presented in the study demonstrate that the 3D UKF-UI-WGI procedure is a powerful tool for structural 
TABLE 3: Stiffness parameter identification of 3D three-story frame.

\begin{tabular}{|c|c|c|c|c|c|c|c|}
\hline \multirow{3}{*}{ Member } & \multirow{3}{*}{$\begin{array}{l}\text { Actual }(k) \\
(\mathrm{kN}-\mathrm{m})\end{array}$} & \multirow{2}{*}{\multicolumn{2}{|c|}{$\begin{array}{c}\text { 3D UKF-UI-WGI } \\
24 \text { DDOFs }\end{array}$}} & \multicolumn{4}{|c|}{ 3D GILS-EKF-UI } \\
\hline & & & & \multicolumn{2}{|c|}{24 DDOFs } & \multicolumn{2}{|c|}{27 DDOFs [2] } \\
\hline & & Identified $(k)$ & Change (\%) & Identified $(k)$ & Change (\%) & Identified $(k)$ & Change (\%) \\
\hline (1) & (2) & (3) & (4) & (5) & (6) & (7) & (8) \\
\hline$k_{1}$ & 13476 & 13515 & 0.29 & 13562 & 0.64 & 13532 & 0.41 \\
\hline$k_{2}$ & 13476 & 9343 & -30.67 & 9288 & -31.08 & 9209 & -31.66 \\
\hline$k_{3}$ & 13476 & 13124 & -2.61 & 11529 & -14.45 & 13647 & 1.27 \\
\hline$k_{4}$ & 13476 & 13529 & 0.39 & 12461 & -7.53 & 13458 & -0.14 \\
\hline$k_{5}$ & 13476 & 13263 & -1.58 & 15358 & 13.96 & 13317 & -1.18 \\
\hline$k_{6}$ & 13476 & 13837 & 2.68 & 15706 & 16.54 & 14680 & 8.93 \\
\hline$k_{7}$ & 13476 & 13452 & -0.18 & 13498 & 0.16 & 13425 & -0.38 \\
\hline$k_{8}$ & 13476 & 13541 & 0.48 & 13359 & -0.87 & 13633 & 1.16 \\
\hline$k_{9}$ & 13476 & 13439 & -0.28 & 14422 & 7.02 & 13479 & 0.02 \\
\hline$k_{10}$ & 13476 & 13507 & 0.23 & 13519 & 0.32 & 13244 & -1.72 \\
\hline$k_{11}$ & 13476 & 13380 & -0.72 & 14110 & 4.70 & 13964 & 3.62 \\
\hline$k_{12}$ & 13476 & 13550 & 0.55 & 12332 & -8.49 & 13285 & -1.42 \\
\hline$k_{13}$ & 14553 & 14530 & -0.16 & 14601 & 0.33 & 14491 & -0.43 \\
\hline$k_{14}$ & 14553 & 14478 & -0.51 & 14500 & -0.36 & 14363 & -1.31 \\
\hline$k_{15}$ & 14553 & 14582 & 0.20 & 13924 & -4.32 & 14557 & 0.03 \\
\hline$k_{16}$ & 14553 & 14272 & -1.93 & 13468 & -7.46 & 13863 & -4.74 \\
\hline$k_{17}$ & 14553 & 14510 & -0.30 & 14579 & 0.18 & 14459 & -0.65 \\
\hline$k_{18}$ & 14553 & 14778 & 1.54 & 13932 & -4.27 & 14881 & 2.25 \\
\hline$k_{19}$ & 14553 & 14410 & -0.98 & 13793 & -5.22 & 14604 & 0.35 \\
\hline$k_{20}$ & 14553 & 14456 & -0.67 & 15213 & 4.53 & 14932 & 2.60 \\
\hline$k_{21}$ & 14553 & 14462 & -0.63 & 14157 & -2.72 & 14715 & 1.11 \\
\hline$k_{22}$ & 14553 & 14513 & -0.27 & 14483 & -0.48 & 14162 & -2.69 \\
\hline$k_{23}$ & 14553 & 14780 & 1.56 & 16595 & 14.03 & 14507 & -0.32 \\
\hline$k_{24}$ & 14553 & 14416 & -0.94 & 14429 & -0.85 & 14372 & -1.24 \\
\hline
\end{tabular}

health assessment. It can identify the location and severity of defective member in large infrastructures. It can also locate the defect spot more accurately within a defective member. The UKF-based SHA procedures are better than that of EKFbased procedures proposed earlier by other research team members of the authors.

\section{Conclusions}

The complexity in the health assessment of civil infrastructures evolves over a long period of time. A simple problem can become very complex based on the current needs, sophistication required, and the technological developments. To meet the current needs of locating defect spots and their severity as accurately as possible, infrastructures are represented by finite elements. To increase the implementation potential, the stiffness parameters of all the elements are identified using only noise-contaminated dynamic responses measured at small part of the infrastructures. To extract the required information, Kalman filter concept used by electrical engineers for signal processing is integrated with other numerical schemes. Since the severity of damage will be unknown at the beginning of an inspection process, an unscented Kalman filter concept is developed for highly nonlinear dynamic systems. The basic UKF concept is improved in several ways. Instead of using one long duration time-history in one global iteration, very short duration time-histories are used and multiple global iterations with weight factors are used to locate the defect spot more accurately and efficiently. With the help of two informative examples, the capabilities of 3D UKFUI-WGI are demonstrated. It can identify the location and severity of defect very accurately even in the presence of large nonlinearity. It has the capability of locating defect spot more accurately within a defective element. It is also documented that the UKF-based structural health assessment procedure is much superior to EKF-based procedure, particularly when the presence of severe defect may make the responses highly nonlinear.

\section{Disclosure}

Any opinions, findings, conclusions, or recommendations expressed in this paper are those of the writers and do not necessarily reflect the views of the sponsors. 


\section{Conflicts of Interest}

The authors declare that they have no conflicts of interest.

\section{Acknowledgments}

This study is based on work partly supported by University of Basrah, Iraq, and is partially supported by the National Science Foundation under Grant no. CMMI-1403844.

\section{References}

[1] C. R. Farrar, W. E. Baker, T. M. Bell et al., "Dynamic characterization and damage detection in the I-40 bridge over the Rio Grande," Tech. Rep. LA-12767-MS, Los Alamos National Laboratory Report, 1994.

[2] A. K. Das, Health assessment of three dimensional large structural systems using limited uncertain dynamic response information [Ph.D. thesis], University of Arizona, Tucson, AZ, USA, 2012.

[3] A. Al-Hussein, A novel technique for structural health assessment in the presence of nonlinearity [Ph.D. thesis], University of Arizona, Tucson, AZ, USA, 2015.

[4] B. H. Oh and B. S. Jung, "Structural damage assessment with combined data of static and modal tests," Journal of Structural Engineering, vol. 124, no. 8, pp. 956-965, 1998.

[5] P. H. Vo and A. Haldar, "Health assessment of beams Theoretical formulation and analytical verification," Structure and Infrastructure Engineering, vol. 4, no. 1, pp. 33-44, 2008.

[6] P. H. Vo and A. Haldar, "Health assessment of beamsexperimental verification," Structure and Infrastructure Engineering, vol. 4, no. 1, pp. 45-56, 2008.

[7] D. Wang and A. Haldar, "Element-level system identification with unknown input," Journal of Engineering Mechanics, vol. 120, no. 1, pp. 159-176, 1994.

[8] X. Ling and A. Haldar, "Element level system identification with unknown input with Rayleigh damping," Journal of Engineering Mechanics, vol. 130, no. 8, pp. 877-885, 2004.

[9] M. Katkhuda, R. Martinez, and A. Haldar, "Health assessment at local level with unknown input excitation," Journal of Structural Engineering, vol. 131, no. 6, pp. 956-965, 2005.

[10] R. E. Kalman, "A new approach to linear filtering and prediction problems," Transactions of the ASME-Journal of Basic Engineering, vol. 82, no. 1, pp. 35-45, 1960.

[11] R. E. Kalman and R. S. Bucy, "New results in linear filtering and prediction theory," Journal of Fluids Engineering, vol. 83, no. 1, pp. 95-108, 1961.

[12] D. Wang and A. Haldar, "System identification with limited observations and without input," Journal of Engineering Mechanics, vol. 123, no. 5, pp. 504-510, 1997.

[13] K. Katkhuda and A. Haldar, "A novel health assessment technique with minimum information," Structural Control and Health Monitoring, vol. 15, no. 6, pp. 821-838, 2008.

[14] A. K. Das and A. Haldar, "Health assessment of three dimensional large structural systems-a novel approach," International Journal of Life Cycle Reliability and Safety Engineering, vol. 1, no. 1, pp. 1-14, 2012.

[15] S. J. Julier, J. K. Uhlmann, and H. F. Durrant-Whyte, "A new approach for filtering nonlinear systems," in Proceedings of the American Control Conference, vol. 3, pp. 1628-1632, Seattle, Wash, USA, June 1995.
[16] S. J. Julier, "The scaled unscented transformation," in Proceedings of the American Control Conference, vol. 6, no. CH37301, pp. 4555-4559, IEEE, Anchorage, AK, USA, May 2002.

[17] A. Al-Hussein and A. Haldar, "Novel unscented kalman filter for health assessment of structural systems with unknown input," Journal of Engineering Mechanics, vol. 141, no. 7, Article ID 08215003, p. 04015012, 2015.

[18] A. Al-Hussein and A. Haldar, "Structural health assessment at a local level using minimum information," Engineering Structures, vol. 88, pp. 100-110, 2015.

[19] A. Al-Hussein and A. Haldar, "Structural damage prognosis of three-dimensional large structural systems," Structure and Infrastructure Engineering, vol. 13, no. 12, pp. 1596-1608, 2017.

[20] M. Paz and M. Leigh, Structural Dynamics: Theory and Computation, Klumer Academic Publishers, Massachusetts, MA, USA, 5th edition, 2004.

[21] A. Al-Hussein and A. Haldar, "Unscented Kalman filter with unknown input and weighted global iteration for health assessment of large structural systems," Structural Control and Health Monitoring, vol. 23, no. 1, pp. 156-175, 2016.

[22] M. Hoshiya and E. Saito, "Structural identification by extended Kalman filter," Journal of Engineering Mechanics, vol. 110, no. 12, pp. 1757-1770, 1984.

[23] R. W. Clough and J. Penzien, Dynamics of Structures, Computers and Structures, CA, USA, 3rd edition, 2003.

[24] ANSYS, Computer Software-Version 15.0, The Engineering Solutions Company, Canonsburg, PA, USA, 2014.

[25] R. Martinez-Flores and A. Haldar, "Experimental verification of a structural health assessment method without excitation information," Journal of Structural Engineering, vol. 34, no. 1, pp. 33-39, 2007. 


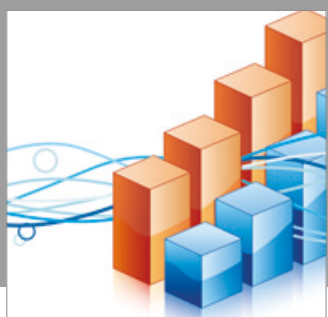

Advances in

Operations Research

vatersals

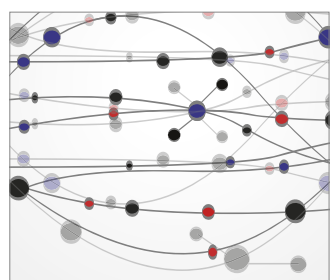

\section{The Scientific} World Journal
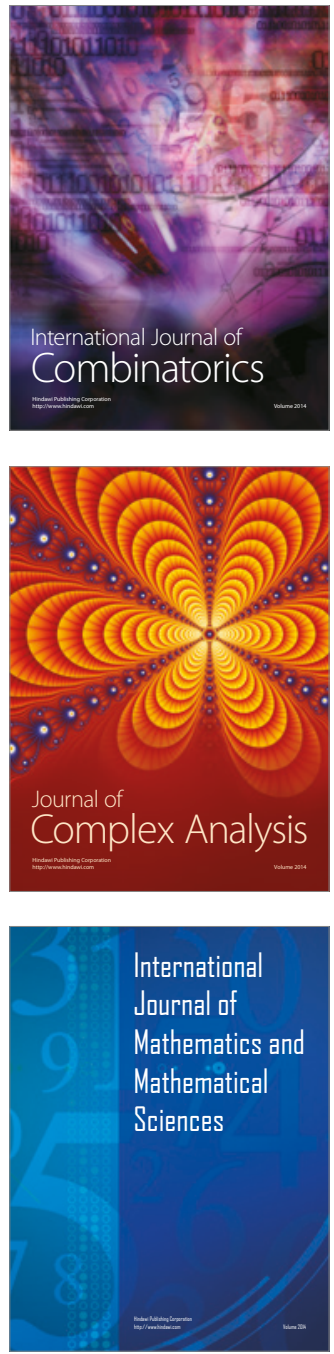
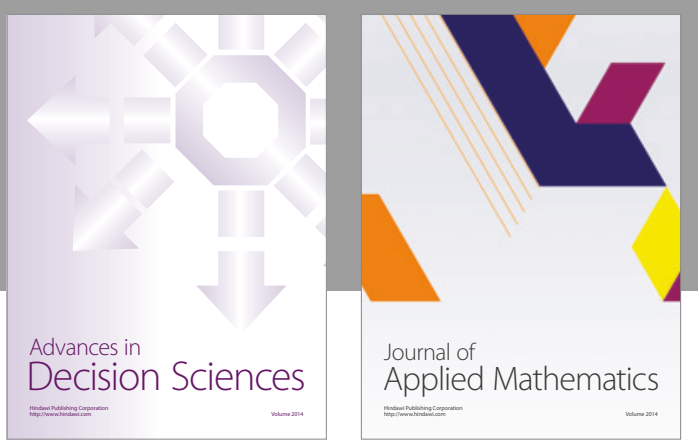

Algebra



\section{Hindawi}

Submit your manuscripts at

https://www.hindawi.com
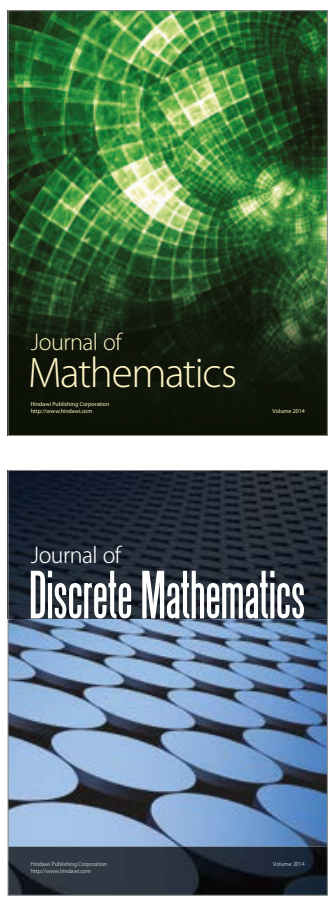

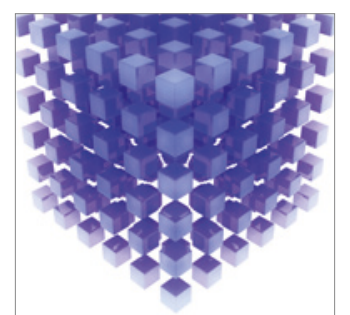

Mathematical Problems in Engineering
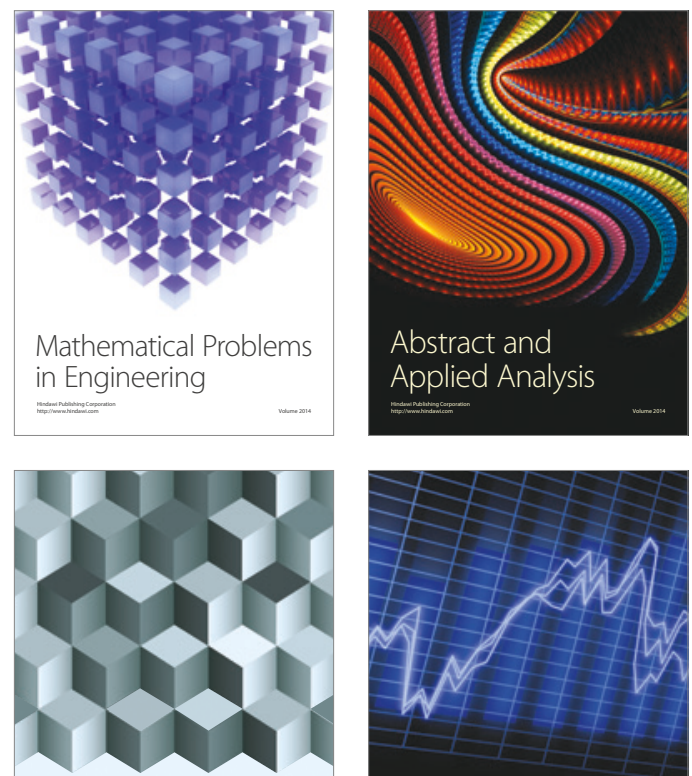

Journal of

Function Spaces

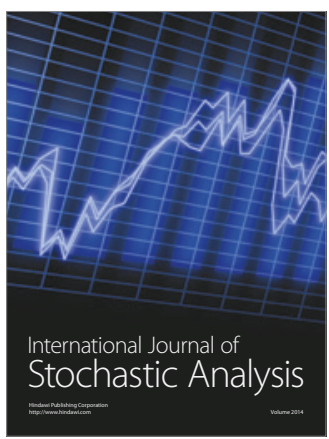

Probability and Statistics
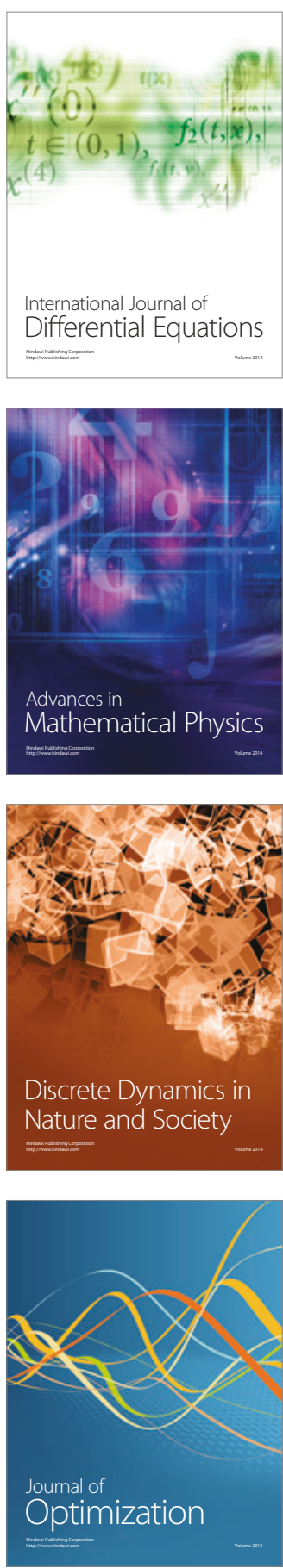